\title{
Helical Structure of Hair Cell Stereocilia Tip Links In the Chinchilla Cochlea
}

\author{
Vladimir Tsuprun and Peter Santi \\ Department of Otolaryngology, University of M innesota, M inneapolis, M N 55455, USA
}

Received: 16 November 1999; Accepted: 15 August 2000; O nline publication: 17 October 2000

\begin{abstract}
O uter-hair-cell stereocilia tip-link structure in the chinchilla cochlea was studied by transmission electron microscopy using tannic acid and Ruthenium red/ Alcian blue histochemical procedures. Tannic acid and Ruthenium red/ Alcian blue treatments showed the tip link as a compact strand of filaments 9-12 nm thick. Fourier analysis of tip-link images showed that the strand is a three-start helical bundle of fine, coiled filaments which had an axial period of $22.5 \pm 1.5 \mathrm{~nm}$. Each of three coiled filaments in the strand showed globular structures, $4.3 \pm 0.3 \mathrm{~nm}$ in diameter. The globular structures may correspond to individual protein subunits or they may be repeating identical domains of one polypeptide. The three filaments of the helical array may provide a rigidity to the tip link during stereocilia deflections. Alternatively, changes in the subunit or domain structure of each filament may result in a lengthening or shortening of the tiplink strand.
\end{abstract}

Keywords: stereocilia, tip link, transmission electron microscopy, image analysis, helical structure

\section{INTRODUCTION}

Deflection of the hair cell stereocilia appears to result in the opening and closing of mechanoelectrical transduction channels which are located at the tips of the stereocilia ( H udspeth and Corey 1977; Coreyand H udspeth 1979, 1983; Howard and Hudspeth 1988). In

Correspondence to: Dr. Peter Santi - University of Minnesota $\cdot \mathrm{Rm}$. 121, Lions Research Building • 2001 Sixth St., SE - Minneapolis, MN 55455. Telephone: (612) 626-9881; fax: (612) 626-9871; email: santip@umn.edu the tip-link model of hair cell transduction, the gating springs correspond to the tip links ( Pickles et al. 1984; H udspeth 1985; Assad et al. 1991; for review, see Pickles and Corey 1992; Garcia-Anveros and Corey 1997). According to this model, stereocilia bundle deflection in the excitatory direction stretches the elastic tip links to open ion channels, while motion in the opposite direction relaxes the tip links and closes the channels (Corey and Hudspeth 1983; Pickles et al. 1984). It has been suggested that mechanoelectrical transduction channels are located at one or both ends of these links (O sborne et al. 1984; Pickles et al. 1984; Denk et al. 1995). However, immunohistochemical experiments with antibodies against the amiloride-sensitive sodium channels (Hackney and Furness 1995) and measurement of current after loss of tip links (Meyer et al. 1998) suggest that the channels may not be located at the end of the tip link. Tip links were also suggested to have an adaptation mechanism, which apparently works by the continuous adjustment of the tension of the tip links by moving the attachment point of the gating spring along the shaft of the longer stereocilium ( for review, see Garcia-Anoveros and Corey 1997). O ne of the possible candidates for the motor protein is myosin I-beta (Howard and Hudspeth 1988; GarciaAnoveros and Corey 1997), which was localized mainly within the cytoplasm of the stereocilium near the upper and lower ends of the tip link by immunoelectron microscopy (Garcia et al. 1998).

Knowledge of the molecular composition and structure of tip links and their interactions with other macromolecules is important for understanding of the mechanoelectrical transduction mechanism in the hair cells. In electron micrographs, the tip links appear as a thin strand of extracellular material that extends from the tip of the shorter stereocilium and attaches to the adjacent, longer stereocilium of the next row 
(O sborne et al. 1984, 1988; Pickles et al. 1984, 1989; Comis et al. 1985; O sborne and Comis 1989; Furness and Hackney 1985; [38] Slepecky and Chamberlain 1985). A darkly stained intracellular plaque was observed where the upper end of the tip link isinserted into the longer stereocilium (Furness and Hackney 1985). Cuprolinic blue revealed electron-dense structures at both ends of the tip link; they may serve to anchor the tip links to the stereocilium membrane (Tsuprun and Santi 1998).

The composition of the tip link has been speculated on but is not yet known. According to Osborne and Comis (1990), the tip link is a filamentous protein surrounded by glycocalyx. Katori et al. (1996) showed, using light and electron microscopy, that a monoclonal antibody against keratan sulfate proteoglycan labeled the tip and side links, as well as the tips of the tallest outer-hair-cell stereocilia, in guinea pigs. H owever, in contrast to the guinea pig, stereocilia bundles of the mouse (Killick and Richardson 1997) and the chinchilla (Swartz and Santi 1997) were not immunolabeled using this antibody. Using immunoelectron microscopy, the presence of type IV collagen in tip links was suggested by $\mathrm{H}$ ackney et al. (1999). H owever, tip-link structural data does not correspond to the thin, interlacing, filamentous arrays formed by the three polypeptide chains of type IV collagen (Kachar et al. 1999). Results from freeze-etching electron microscopy (Kachar et al. 1999) showed that the tip link has diameter of 8-11 nm and consists of protein subunits arranged into a double-helical, right-handed, coiled strand with a 60-nm pitch. In addition, the filaments appear to separate at their ends and form multiple insertion points into the stereocilium membrane.

The purpose of the present study was to investigate the structure of stereocilia tip links in the cochlear sensory epithelium of the chinchilla by transmission electron microscopy using different histochemical reagents. Fourier analysis was applied to the images to verify the presence of the helical structure of the tip-link strand and to study the spatial arrangement of its structural units.

\section{MATERIALS AND METHODS}

Samples of cochlear tissue were obtained from our tissue bank. The samples were from normal chinchillas approximately 1 year old. In order to obtain cochlear tissues for the tissue bank, the animals were deeply anesthetized and their cochleas were removed and perfused with fixatives as previously described (Santi et al. 1990). The care and use of the animals for this study were reviewed and approved by the U niversity of Minnesota's Institutional Animal Care Committee.

Tannic acid and Ruthenium red/ Alcian blue histochemical reagents, in combination with different chemical fixatives, were used for transmission electron microscopy to obtain structural information on stereocilia tip links. Tannic acid reacts with many proteins and glycoproteins, irrespective of their electrical charge ( $\mathrm{H}$ ayat 1989). Ruthenium red/Alcian blue, developed by van Kuppelvelt and coworkers (1984), strongly reacts with anionic sites on glycoconjugates and proteins.

For tannic acid treatment, cochleas from four animals were fixed for $1 \mathrm{~h}$ at $\mathrm{pH} 5.3$ in a solution containing $2 \%$ paraformaldehyde in $0.075 \mathrm{M}$ cacodylate buffer, $4 \mathrm{mM} \mathrm{CaCl} 2,2 \%$ tannic acid, and washed in $0.13 \mathrm{M}$ cacodylate buffer at $\mathrm{pH} 6.8$ for $45 \mathrm{~min}$. The tissues were postfixed in $1 \% \mathrm{O} \mathrm{sO}_{4}$ in $0.1 \mathrm{M}$ cacodylate buffer at $\mathrm{pH} 6.8$ for $1 \mathrm{~h}$ at room temperature and washed in $0.13 \mathrm{M}$ cacodylate buffer for $45 \mathrm{~min}$. For Ruthenium red/ Alcian blue treatment, cochleas from three animals were fixed in a solution of $0.2 \%$ Ruthenium red, $0.2 \%$ Alcian blue, $2.5 \%$ glutaraldehyde in $0.1 \mathrm{M}$ cacodylate buffer for $4 \mathrm{~h}$ and postfixed in $1 \%$ osmium tetroxide for $1 \mathrm{~h}$.

Cochlear tissues were then dehydrated in ascending concentrations of ethanol, embedded in Epon, and sectioned on an LKB Nova ultramicrotome for transmission electron microscopy as previously described (Santi 1986). Thin sections, without counterstaining, were examined using a JEOL 1010 electron microscope at $60 \mathrm{kV}$ and magnifications of 20,000X.

M icrographs were digitized by a L inotype- $\mathrm{H}$ ell flatbed scanner interfaced to a Macintosh computer. The pixel size of the scanned images was about $1.4 \mathrm{~nm}$ at the specimen level. Some images were filtered using a high-pass filter via Adobe Photoshop software in order to suppress the very low spatial frequencies (1/ $10 \mathrm{~nm}^{-1}$ and below) and, thus, to increase the signalto-noise ratio of the images. For these purposes, the Fourier transform of the image was multiplied by a function of a filter passing high frequencies. The low frequencies, which normally do not contribute to the structure, were set to reduced values (Russ 1999). Noise filtering and adjustment of the image brightness and contrast of the entire image field were used to enhance the images and accentuate their details. To study the periodic helical structure of the tip links, their digitized images were Fourier transformed. The theory of Fourier transform relevant to the helical diffraction patterns was covered in Cochran et al. (1952) and Klug et al. (1958), while its application to electron micrographs was discussed by DeRosier and Moore (1970). NIH Image (ver. 1.60) software (NIH, Bethesda, MD) was used for the Fourier transformation ( $256 \times 256$ pixel size). The selected area of the 


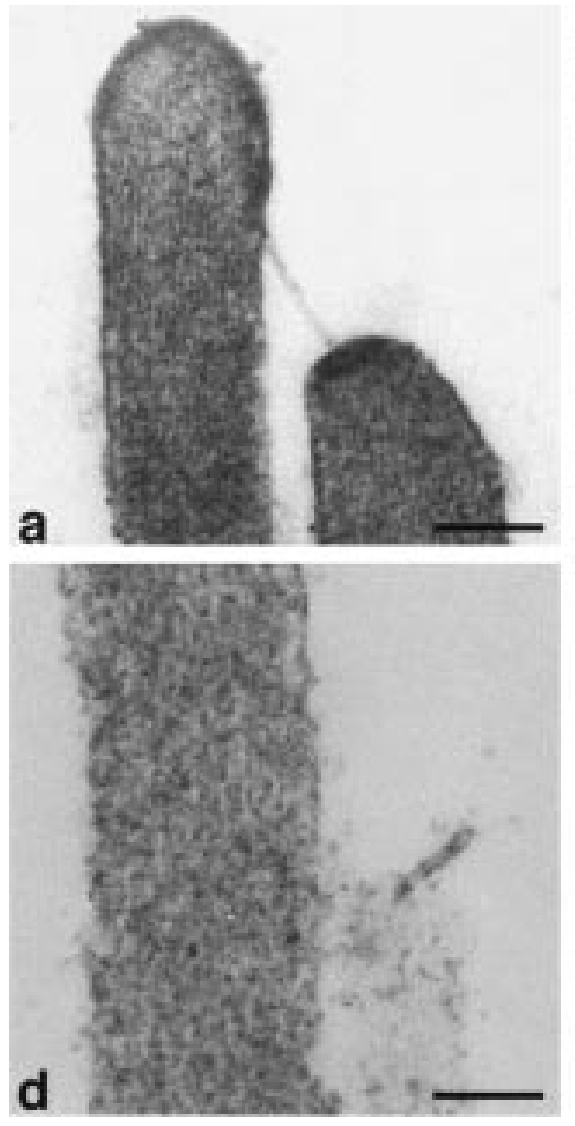

FIG. 1. TEM of tip links after tannic acid treatment. a, d Micrographs of adjacent stereocilia connected by the tip links. b, e Noise-filtered images corresponding to $\mathbf{a}$ and $\mathbf{d}$, respectively. $\mathbf{c}$, f High-power images of the tip links shown in $\mathbf{b}$ and $\mathbf{e}$, respectively, and computer Fourier

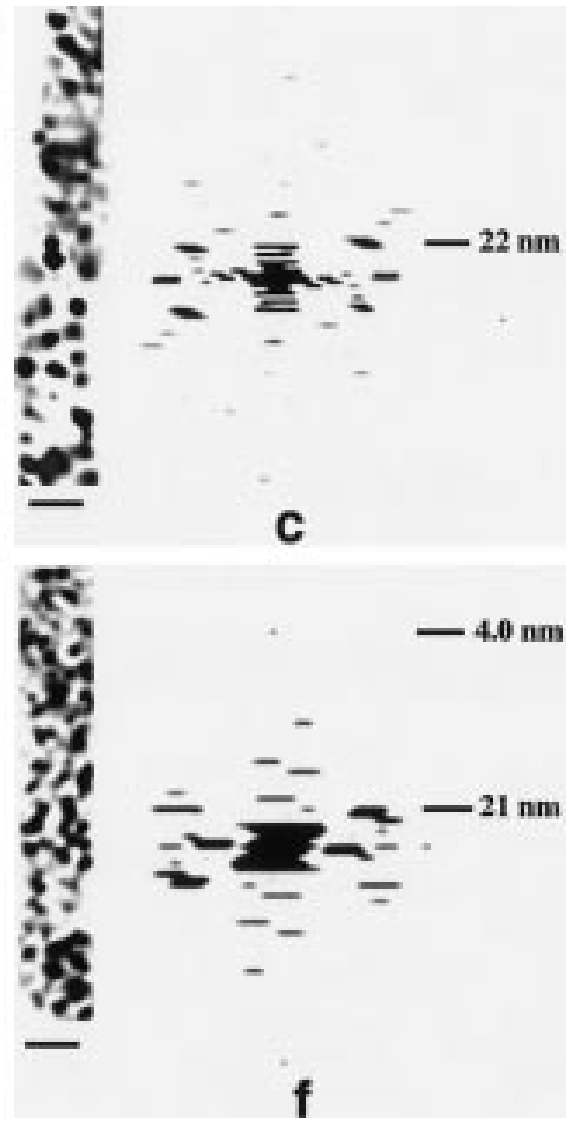

transforms from their rectangular areas. Two strong diffraction peaks and one weaker peak corresponds to a periodicity of 21-22 nm and about $4.0 \mathrm{~nm}$, respectively. Bars $=100 \mathrm{~nm}(\mathbf{a}, \mathbf{b}, \mathbf{d}, \mathbf{e})$ and $10 \mathrm{~nm}$ $(\mathbf{c}, \mathbf{f})$. tip-link image, smaller than 256 pixels, was brought up to a standard $256 \times 256$ size box with addition of densities of zero magnitude outside the image. The transform sampling interval was $(1.4 \times 256)^{-1} \mathrm{~nm}^{-1}$ in the $X$ and $Y$ directions. The periodicity of the structure was calculated from the position of diffraction peaks of the Fourier transform of the image with known periodicity and the magnification of the print for calibration. The quality and reproducibility of tiplink images were analyzed from their Fourier diffraction patterns based on the presence of characteristic intensities of about 20-nm periodicity and image resolution. The fine structure of the tip links was not usually well preserved. About 20 tip-link images were analyzed. Most of these images did not show strong diffraction peaks, and only seven of tip-link images (three from tannic acid and four from Ruthenium blue/ Alcian blue treatments) showed strong symmetric intensities corresponding to a periodicity of about $20 \mathrm{~nm}$. Four of these images (two from tannic acid and two from Ruthenium red treatments) demonstrated weak, high-resolution meridianal intensity of about 4$\mathrm{nm}$ periodicity.

\section{RESULTS}

The tip links were observed in radial sections of cochlear hair cells across all three rows of the outer hair cells. After tannic acid treatment (Fig. 1a-f), the tip link appeared as a thin straight strand with a typical length of 150-200 nm. It extended between the tip of shorter stereocilium to the side wall of the longer stereocilium (Fig. 1a). A darkly stained intracellular plaque was visible near the lower end of the link beneath the membrane of the shorter stereocilium (Fig. 1a). The upper end of the link joined another darkly stained intracellular plaque associated with the membrane of the longer stereocilium (Fig. 1a). In rare images, one end of the tip link was detached from the stereocilium membrane (Fig. 1d). Some high-passfiltered images of tip links ( see M aterialsand M ethods) show small rounded particles (Fig. 1c,f). To study the periodic distribution of these particles, the noise-filtered images of the tip links were Fourier transformed (see Materials and Methods). In high-quality Fourier transforms of the tip-link images ( Fig. 1c,f), two strong 

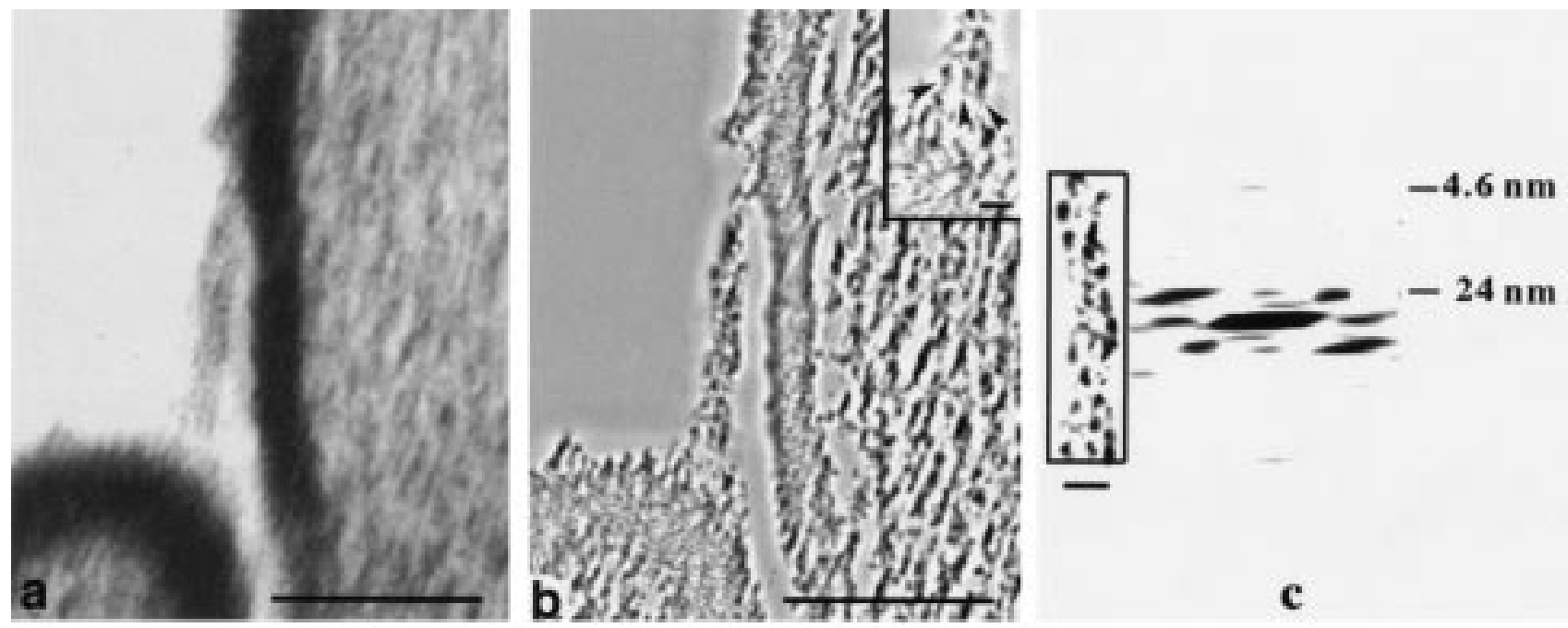

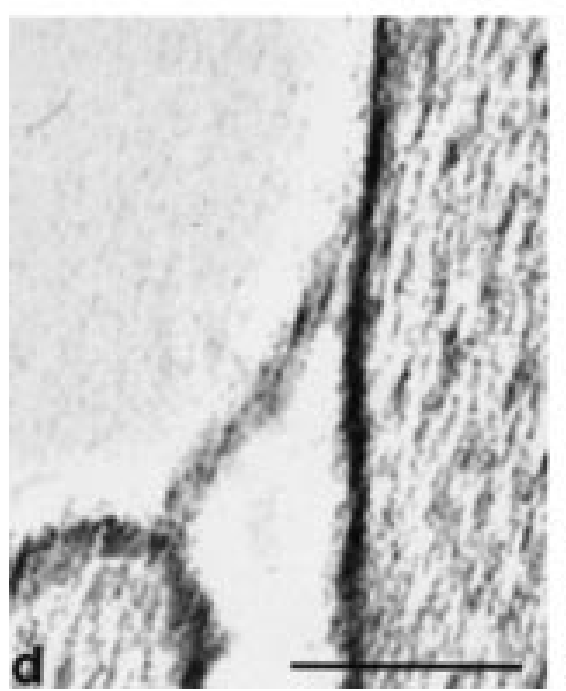

FIG. 2. TEM of tip links after Ruthenium red/Alcian blue treatment. a, d Micrographs of adjacent stereocilia connected by the tip links. b, e Noise-filtered images corresponding to $\mathbf{a}$ and $\mathbf{d}$, respectively. Three fine filaments (arrowheads) of the tip links are shown in inset of $\mathbf{b}$ and e. $\mathbf{c}, \mathbf{f}$ High-power images of the tip-link filamentous bundles symmetric peaks are located on the layer line corresponding to a periodicity of 21-22 nm (Fig. 1C,f). A weak peak is sometimes seen on the layer line corresponding to a periodicity of about $4.0 \mathrm{~nm}$ (Fig. 1f).

Ruthenium red/ Alcian blue treatment (Fig. 2) provided a staining pattern of tip links similar to that observed after tannic acid treatment. The tip link showed a thin strand 9-12 $\mathrm{nm}$ in diameter. Three filaments were occasionally observed in these strands (Fig. 2b,e, inset). After high-pass filtration of the tiplink images, a bundle of fine "beaded filaments" can be distinguished in the high-power images (Fig. 2c,f). Fourier transforms of the bundle images (Fig. 2c,f) show two strong diffraction peaks located on the 2324-nm layer line and a much weaker meridian intensity on the 4.3-4.6-nm layer line. This distribution of diffraction intensities was similar to those observed after tannic acid treatment (Fig. 1c,f).

For control and calibration of observed periodicities, Fourier transformation was performed on actin filaments in the core of the stereocilia taken from the same micrographs that were used for the tip-link analysis (Fig. 3a-c). Actin filamentsare packed in paracrystalline arrays with aligned crossover points of all actin helices. Diffraction patterns from these arrays showed strong equatorial peaks corresponding to $11 \pm 1$-nm periodicity between actin filaments, and two symmetric peaks on the 35-nm layer line corresponding to the periodicity of crossover points of Factin in the stereocilia (Tilney et al. 1980). 


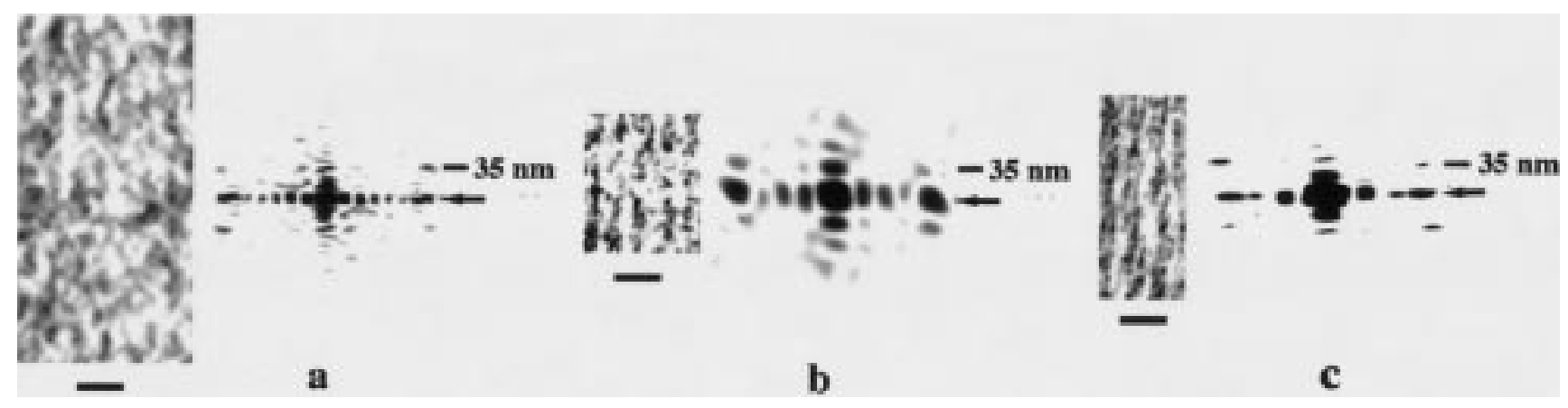

FIG. 3. a-c Computer diffraction of F-actin paracrystalline arrays in thin stereocila sections shows two equatorial peaks (arrows) corresponding to regular repeat between actin filaments and two symmetric peaks on the 35-nm layer line corresponding to periodicity of the crossover points of F-actin. Images of the F-actin arrays are taken from the micrographs shown in 1b, 2b, and 2e, respectively. Bars $=20 \mathrm{~nm}$.

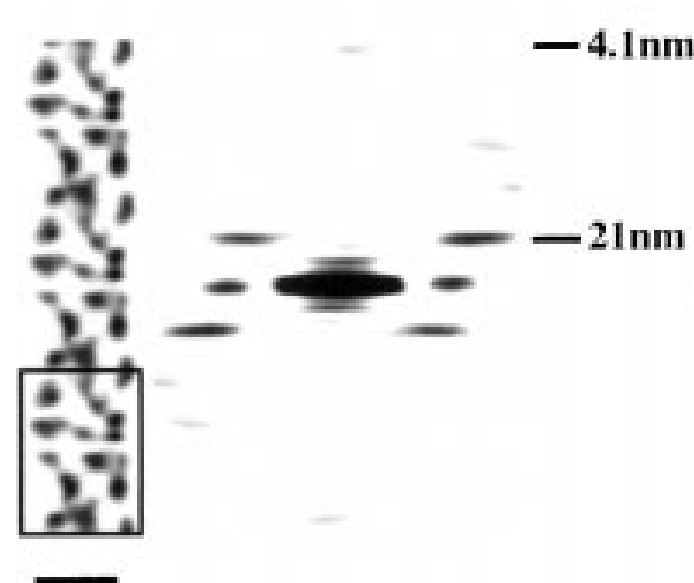

a

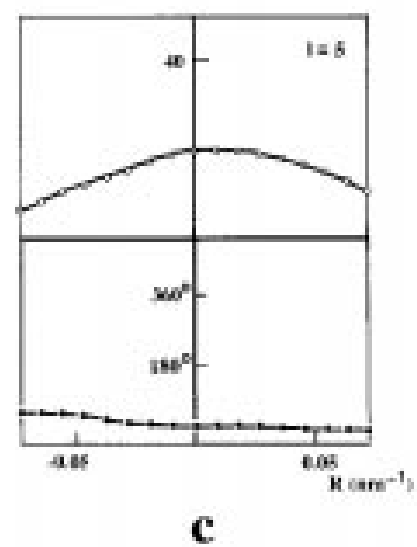

FIG. 4. a Computer-averaged image composed of three identical fragments. Each fragment (rectangle) was obtained by digital averaging over one axial period $(21 \mathrm{~nm})$ of the tip-link image shown in Fig. 1f. Fourier transform from this image is similar to the diffraction pattern of the original image (Fig. 1f). Bar $=10 \mathrm{~nm}$. Amplitudes and phases for of 21-nm $(I=1)$ and 4-nm $(I=5)$ layer lines are shown in $\mathbf{b}$ and $\mathbf{c}$, respectively. The phases corresponding to two peaks

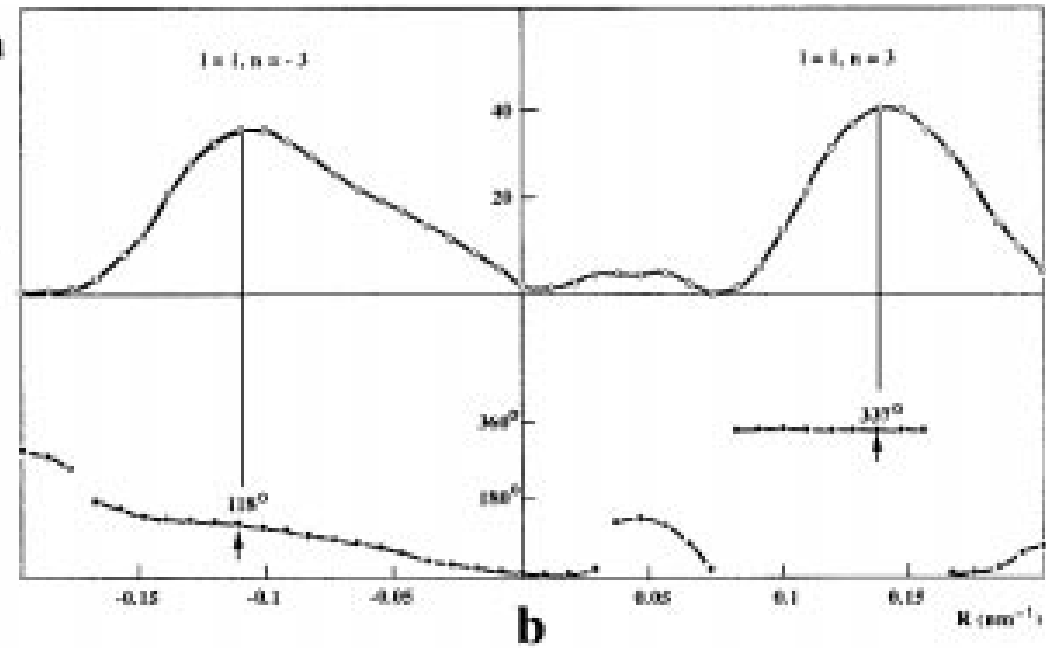

$-4.4 \mathrm{~nm}$
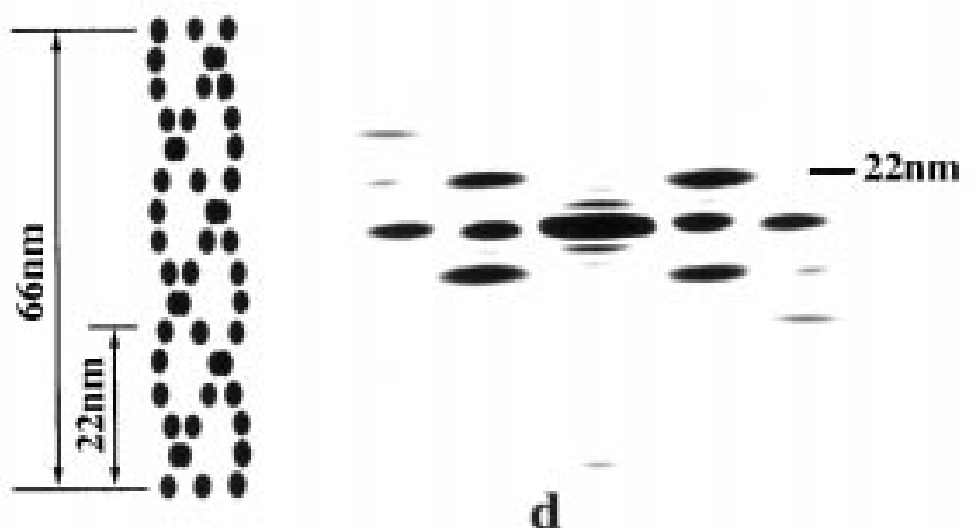

at 21-nm layer line are arrowed. d Projected structure and Fourier transform of the tip-link model. Three coiled helices of 15 globular units per $66-\mathrm{nm}$ pitch are related by threefold rotational symmetry. This symmetry results in 22-nm axial period of the helical array observed in the Fourier transforms. Meridional diffraction peak of 4.4-nm periodicity corresponds axial shift of globular units. 
As an additional control for the $22.5 \pm 1.5-\mathrm{nm}$ axial period of the tip link observed in the diffraction pattern, one of the tip-link images (Fig. 1e) was computer averaged over this period. The image was displayed at high magnification, and its repeating portions, which corresponded to one period ( $21 \mathrm{~nm}$ ), were selected by a 21-nm shift of the rectangular frame along the helical axis. The set included three of these images, each $21 \mathrm{~nm}$ high, and was converted to a stack by NIH Image software and arithmetically averaged (Fig. 4a, rectangle). The final image was composed from three of these averaged portions (Fig. 4a). Diffraction pattern from this image was similar to the original image (Fig. 1f) and showed two strong peaks on the 21-nm layer line (Fig. 4a). Computer averaging also resulted in noise reduction of the image. Symmetric location of diffraction peaks relative to the meridian on horizontal layer lines is characteristic for a structure with helical symmetry and each layer line arising from a particular family of helices in the structure (Cochran et al. 1952; Klug et al. 1958; DeRosier and Moore 1970). The position of each peak on layer lines is related to the Bessel function $\mathscr{T}_{n}(2 \pi R)$, where $r$ is the helix radius. The relative phase of the two peaks shows whether $n$ (number of helices) is odd or even. If the phase origin is on the helical axis, two peaks should be in phase for an even value of $n$ or out of phase for an odd value of $n$. Two symmetric peaks on the 21-nm $(I=1)$ layer line were derived from the near and far sides of the tip-link helical structure. Image-Pro Plus (version 4.1 for Windows, Media Cybernetics, Silver Springs, MD) software was used for the calculation of the amplitudes and phases of the Fourier transform of the tip-link images. Figure $4 \mathrm{~b}$ shows the computed amplitudes and phase on the 21-nm layer line. The position of the two peaks on this layer line is such that $n$ is between 2 and 4. The computed phases of these peaks differ by about $219^{\circ}$ or, with some approximation, are out of phase. This reduces this choice to an odd number, or $n=3$, of the helical filaments in the tip link. Occasionally, these three filaments can be directly visible in some tiplink images (Fig. 2b,e, inset). The weaker diffraction intensity on the 4.1-nm layer line $(l=5)$ is located around the meridian. This intensity shows only one peak with a small variation of phase (Fig. 4c) and appears to correspond to the zero order of the Bessel function. [Thus, a tentative model of the tip link having a three-start helical structure is proposed to approximate these diffraction data (Fig. 4d).] The model consists of three filaments with 15 globular units per 66-nm pitch of each filament. Since three helices of the 66-nm pitch are related by threefold rotational symmetry (by $120^{\circ}$ rotation), the period of the helical structure is $22 \mathrm{~nm}$. The diffraction pattern from the projected structure of the model shows two symmetrical peaks located on a 22-nm (I = 1) layer line and a 4.4-nm ( $=5)$ meridian peak which correspond to an axial period of the helical array and axial shift of globular units, respectively. Diffraction peaks of the first and second order of the 66-nm periodicity were not seen.

\section{DISCUSSION}

According to the "gating spring" model, increased tension in the gating spring associated with the tip link opens transduction channels which permits cations to enter the stereocilium and depolarize the cell. M olecular composition and assembly of the tip-link helical structure, and, ultimately, the molecular basis of its elastic behavior, are still unknown. The helical structure of the tip link is stabilized by a large number of contacts between repeating structural units of its three coiled filaments. The helical array may provide a stable nonflexible structure that acts as a rigid spring during hair cell mechanical stimulation and relaxation.

The first report of a tip-link strand showing a righthanded, coiled double filament that ends in two insertion points on the membrane of the taller stereocilia and three insertion points on the membrane of the short stereocilia was provided by Kachar et al. (1999) using freeze-etching electron microscopy. Our data agree with their report concerning the helical organization of the tip link and extends their findings using two different histochemical procedures and Fourier image analysis. In contrast to the Kachar et al. (1999) results, our data show that the tip link is a three- not a two-start coiled filament. Tannic acid and Ruthenium red/ Alcian blue treatments showed that the tip link consists of a straight strand of fine filaments attached to the stereocilia membrane. Fourier analysis of the tip-link strand revealed that this strand is a three-start helical bundle of fine filaments with a period of $22.5 \pm 1.5 \mathrm{~nm}$. Each of the three coiled filaments actually consists of identical globular structures, $4.3 \pm$ $0.3 \mathrm{~nm}$ in diameter, arranged into a helix with about 15 structures per $67.5 \pm 4.5 \mathrm{~nm}$ turn. The $67 \pm 4.5$ $\mathrm{nm}$ pitch of individual filaments can be traced in some tip-link images (Fig. 2c,f and Fig. 4a), and appears to correspond to $60-\mathrm{nm}$ period of right-handed helical filaments as reported by Kachar et al. (1999). Tip links are reactive to tannic acid and appear to contain predominantly neutral or basic residues of proteins. Taking into account the typical $V /$ r ratio of $1.2-1.4 \mathrm{~nm}^{3} /$ $\mathrm{kDa}$ for protein macromolecules, the globular structure of the tip link is a spherical particle with a diameter of about $4.3 \mathrm{~nm}$ and has a molecular weight of approximately $30-35 \mathrm{kDa}$. This corresponds to a polypeptide chain containing approximately 300 amino acids folded into a compact globule (Metzler 1977).

Two possible variants for the interpretation of these 
globular structures observed in the tip link can be considered. The globular particles may correspond to individual protein subunits periodically arranged into three coiled filaments. The assembly of the helical structure from protein subunits correlates with $\mathrm{Ca}^{2+}$. dependent stability of the links. $\mathrm{Ca}^{2+}$ chelators are likely to disrupt the tip links by their depolymerization resulting in a change in transduction channel responsiveness (Assad et al. 1991; Marquis and Hudspeth 1997; Meyer et al. 1998). Stretching of the tip links from two to three times their resting length was suggested from previous physiological experiments (Shepherd and Corey 1994). However, a helical array of identical subunits, stabilized by many repeating subunit-subunit interactions along each filament and between neighboring filaments, would feature low elasticity and stiffness. Nevertheless, in some cases of conformational changes of subunits, helical structure may undergo to great variation of its length. This process was described in detail for the contractile tail of T-even bacteriophages (Moody 1973; Amos and Klug 1975).

An alternative interpretation of the data is that each filament of the tip-link bundle consists of one polypeptide of identical domains of this polypeptide folded in regularly arranged globular structures. A similar structural organization was previously reported for titin, a giant filamentous protein from muscle (Trinick et al. 1984). The titin molecule consists of copies of modules, which are thought to belong to fibronectin type III and immunoglobulin protein superfamilies, with the modules arranged into repeating motifs. The titin filament was shown from electron micrographs to consist of linear arrays of 4.3-nm globular domains, with each motif folding into a separate domain (Trinick et al. 1984; Tskhorebova and Trinick 1997). Titin molecules can be stretched to more than 10 times their resting length. This process may unfold the fibronectin or immunoglobulin modules (Tskhorebova and Trinick 1997; Paci and Karplus 1999). The titin-like domain structure was suggested earlier to be responsible for considerable stretching of the tip-link strand (Garcia-Anoveros and Corey 1997). This structure can be attributed to the individual filaments of the helical strand. It would allow an elongation of the whole strand, whereas lateral contacts between filaments would result in the tip-link rigidity.

\section{ACKNOWLEDGMENT}

This research was supported by grants from the NIDCD, including a fellowship grant (NIDCD F32 DC00313-03) to Tsuprun.

\section{REFERENCES}

Amos LA, KLU A A. Three-dimensional image reconstruction of the contractile tail of T4 bacteriophage. J. Mol. Biol. 99:51-64, 1975.

AsSAd JA, Shepherd GM, Corey DP. Tip-link integrity and mechanical transduction in vertebrate hair cells. Neuron. 7:985-994, 1991.

COCHRAN W, CRICK FH C, VAND V. The structure of synthetic polypeptides. I. Transform of atoms on a helix. Acta Crystallogr. 5:581586, 1952, DOI:10.1107/ S0365110X 52001635.

COMIS SD, PICKLES JO, O SBORNE MP. O smium tetroxide postfixation in relation to the cross linkage and spatial organization of stereocilia in the guinea-pig cochlea. J. N eurocytol. 14:113-130, 1985.

COREY DP, HUDSPETH AJ. Ionic basis of the receptor potential in vertebrate hair cell. Nature. 281:675-677, 1979.

COREY DP, HUdSPETH AJ. Kinetics of the receptor current in bullfrog saccular hair cells. J. Neurosci. 3:962-976, 1983

Denk W, Holt JR, Shepherd GM, CoRey DP. Calcium imaging of single stereocilia in hair cells: localization of transduction channels at both ends of tip links. Neuron. 15:1311-1321, 1995.

DeRosier DJ, Moore PB. Three dimensional images from electron micrographs of structures with helical symmetry. J. Mol. Biol. 52:355-359, 1970.

FURNESS DN, HACKNEY CM. Cross-links between stereocilia in the guinea pig cochlea. Hear. Res. 18:177-188, 1985.

Garcia-Anoveros J, Corey DP. The molecules of mechanosensation. Ann. Rev. Neurosci. 20:567-594, 1997.

Garcia JA, YeE AG, GILLESPIE PG, Corey DP. Localization of myosinI $\beta$ near both ends of tip links in frog saccular hair cells. J. Neurosci. 18:8637-8647, 1998.

H ACKNEY CM, FURNESS DN. Mechanotransduction in vertebrate hair cells: structure and function of the stereocilia bundle. Am. J. Physiol. 268:C1-C13, 1995.

HACKNEY CM, WEST B, FURNESS DN. Localization of collagen IV in the guinea-pig organ of Corti using post-embedding immunogold labeling: Do the tip links contain collagen IV? Assoc. Res. Otolaryngol. Abstr. Abstr. 17:4, 1999.

H AYAT MA. Principles and Techniques of Electron microscopy. Biological applications. CRC Press Boca Raton, FL, 1989.

H OWARD J, HUDSPETH AJ. Compliance of the hair bundle associated with gating of mechanoelectric transduction channels in the bullfrog's saccular hair cell. Neuron. 1:189-199, 1988.

HUdSPETH AJ, CoRey DP. Sensitivity, polarity, and conductance change in the response of vertebrate hair to controlled mechanical stimuli. Proc. Nat. Acad. Sci. USA. 74:2407-2411, 1977.

HUDSPETH AJ. The cellular basis of hearing: the biophysics of hair cells. Science. 230:745-752, 1985.

Kachar B, Frolenkov G, Mammano F, Belyantseva I. Freezeetching structural analysis of macromolecular assemblies involved in the outer hair cell mechanoreceptor function. Proc. Sendai Ear Symp. 9:12-13, 1999.

KATORI Y, HACKNEY CM, FURNESS DN. Immunoreactivity of sensory hair bundles of the guinea-pig cochlea to antibodiesagainst elastin and keratan sulphate. Cell Tissue Res. 284:473-479, 1996.

KILLICK R, RICHARDSO N GP. Antibodies to the sulphated, high molecular mouse tectorin stain hair bundles and the olfactory mucus layer. Hear. Res. 103:131-141, 1997, DOI:10.1016/ S0378-5955, 96, 00174-8.

KLUg A, CRICK FHC, Wyckoff HW. Diffraction by helical structures. Acta Crystallogr. 11:199-213, 1958, DOI: 10.1107/ S0365110X 58000517.

MARQUIS RE, HUDSPETH AJ. Effects of extracellular $\mathrm{Ca}^{2+}$ concentration on hair-bundle stiffness and gating-spring integrity in hair cells. Proc. Nat. Acad.Sci. USA. 94:11923-11928, 1997.

Metzler D. Biochemistry. Academic Press, New York 1977.

Meyer J, Furness DN, Zenner HP, Hackney CM, Gummer AW. 
Evidence for opening of hair-cell transducer channels after tiplink loss. J. Neurosci. 18:6748-6756, 1998.

Moody MF. Sheath of bacteriophage T4. Contraction mechanism deduced from partially contracted sheaths. J. Mol. Biol. 80:613636, 1973.

OSBORNE MP, COMIS SD, PICKLES JO . Morphology and cross-linkage of stereocilia in the guinea-pig labyrinth examined without the use of osmium as a fixative. Cell Tissue Res. 237:43-48, 1984.

OSBORNe MP, COMIS SD, PICKLES JO. Further observations of the fine structure of the tip links between stereocilia of the guinea pig cochlea. Hear. Res. 35:99-108, 1988.

OSBORNE MP, COMIS SD. Action of elastase, collagenase and other enzymes upon linkages between stereocilia in guinea-pig cochlea. Acta Otolaryngol. 110:37-45, 1990.

PACI E, KARPLUS M. Forced unfolding of fibronectin type 3 modules: an analysis by biased molecular dynamics simulation. J. Mol. Biol. 283:441-459, 1999.

PickLes JO, Comis SD, O sBorne MP. Cross-links between stereocilia in the guinea pig organ of Corti, and their possible relation to sensory transduction. Hear. Res. 15:103-112, 1984.

Pickles JO, Brix J, Comis SD, Gleich O, Koppl C, Manley GA, O SBORNE MP. The organization of the tip links and stereocilia on hair cells of bird and lizard basilar papillae. Hear. Res. 41:3141, 1989.

PICKLES JO, COREY DP. Mechanoelectrical transduction by hair cells. Trends Neurosci. 15:254-259, 1992.

Prieto JJ, Merchan JA. Tannic acid staining of the cell coat of the organ of Corti. Hear. Res. 24:237-241, 1986.
Russ JC. The Image Processing Handbook. CRC Press, Raleigh, NC, 1999.

SANTI PA. Organ of Corti surface preparation for computer-assisted morphometry. Hear. Res. 24:179-187, 1986.

SANTI PA, LEASE MK, HARRISON RG, WiCKLER EM. Ultrastructure of proteoglycans in the tectorial membrane. J. Electron Microscope Tech. 15:293-300, 1990.

SHEPHERD G, COREY DP. The extent of adaptation in bullfrog saccular hair cells. J. Neurosci. 14:6217-6229, 1994.

SlepeCky N, Chamberlain SC. The cell coat of inner ear sensory and supporting cells as demonstrated by ruthenium red. Hear. Res. 17:281-288, 1985.

SWARTZ DJ, SANTI PA. Immunohistochemical localization of keratan sulfate in the chinchilla inner ear. Hear. Res. 109:92-101, 1997, DOI:10.1016/ S0378-5955, (97), 00054-3.

TILNEY LG, DEROSIER DJ, MulRoy MJ. The organization of actin filaments in the stereocilia of the cochlear hair cells. J. Cell Biol. 86:244-259, 1980.

Trinick J, KNIGHT P, Whiting A. Purification and properties of native titin. J. Mol. Biol. 180:331-356, 1984.

TSKHOREBOVA L, TRINICK J. Direct visualization of extensibility in isolated titin molecules. J. Mol. Biol. 265:100-106, 1997, DOI: 10.1006/ jmbi.1996.0717.

TSUPRUN V, SANTI P. Structure of outer hair cell stereocilia links in the chinchilla. J. Neurocytol. 27:517-528, 1998.

Van Kuppelvelt TH, Domen JG, Cremers FP, Kuyper CM. Staining of proteoglycans in mouse lung alveoli. I. U Itrastructural localization of anionic sites. Histochem. J. 16:657-659, 1984. 